\title{
Influence of Ground Conductivity on the Over Voltages Induced on Overhead Power Distribution Lines due to an Indirect Lightning Stroke
}

\author{
P.Durai Kannu and Joy Thomas $M$. \\ Department of High Voltage Engineering \\ Indian Institute of Science \\ - Bangalore 560012, INDIA.
}

\begin{abstract}
Computation of induced voltage on an overhead line due to an indirect lightning stroke involves calculation of electric field due to the lightning: stroke as well as its coupling with the overhead line.' The ground conductivity influences both the electric field as well as the coupling mechanism and hence the magnitude and waveshape of the induced voltage. In this paper, lightning induced voltages are calculated for $33 \mathrm{kV}, 3 \phi$ single circuit and double circuit over head power distribution lines. It has been observed that the magnitude and wave shape of the induced voltage is influenced by the finite ground conductivity as well as the number of over head conductors in a multiconductor system.
\end{abstract}

\section{Introduction}

Induced over voltages due to nearby lightning is one of the main causes of medium voltage power distribution line outages and temporary upset or permanent damage to substation electronic control systems. The main steps in computing the lightning induced voltage are

1. Modeling the lightning return stroke.

2. Estimating the fields generated by the lightning including the distortions introduced by the finitely conducting ground.

3. Modeling the field to overhead conductor coupling including the influence of ground impedance.

The ground conductivity plays a role both in the distortions of radiated field as well as in coupling of field to overhead line. The aim of this paper is to study the effect of ground conductivity on the magnitude and waveshape of the induced voltage on overhead power distribution lines. Earlier authors included the effect of ground conductivity in the computation of lightning induced voltages by considering the ground conductivity both in the calculation of horizontal component of electric field as well as in coupling mechanism either individually or together[1-4]. This paper gives the results of the induced voltage due to a nearby lightning for $33 \mathrm{kV}$, $3 \phi$ single circuit and double circuit distribution lines for i) assuming ground as a perfect conductor ii) considering the conductivity of ground only in the calculation of horizontal electric field and iii) including the ground conductivity in the calculation of horizontal electric field and in the field to conductor coupling. The influence of the presence of other conductors on the induced voltage in a multiconductor system is also studied.

Different approximations have been used for including the influence of ground impedance in the calculation of horizontal electric field as well as in the coupling of field to the overhead conductors. In the time domain coupling equations used for the calculation of induced voltage, the frequency dependent ground impedance is represented by a convolution integral. The low frequency approximation used for the transient ground resistance presents singularity as $t \rightarrow 0$. In this paper for the solution of convolution integral a new numerical expression has been used which circumvents the singularity.

\section{The Analysis}

\section{A. Lightning Return Stroke Model}

The model adopted for lightning return stroke is the modified transmission line (MTL) model. In this model it is assumed that the current waveform at the ground decreases exponentialy with height as it travels up the lightning channel at a constant velocity $v$. Mathematicaly this current $i\left(z^{\prime}, t\right)$ at height $z^{\prime}$ and at time $t$ is represented by

$$
i\left(z^{\prime}, t\right)=e^{\left(-z^{\prime} / \lambda\right)} i\left(0, t-z^{\prime} / v\right)
$$

where $\lambda$ is the decay constant to account for the effect of the vertical distribution of charge stored in the corona sheath of the leader and subsequent discharge during the return stroke phase.

$i(0, t)$ is the lightning return stroke current at ground level (channel base current). In this paper the induced voltages are computed for a channel base current of $12 \mathrm{kA}$ peak and maximum time derivative of $40 \mathrm{kA} / \mu s$. The return stroke velocity $v$ is taken as $1.3 \times 10^{8} \mathrm{~m} / \mathrm{s}$ [5]. The lightning channel is assumed as straight and vertical.

\section{B. Electric Fields generated by the Return Stroke}

By assuming that the ground is a perfect conductor, the vertical component of electrical field $d E_{z}(r, z, t)$ and the horizontal component of electric field $d E_{r}(r, z, t)$ due to an infinitesimal length $d z^{\prime}$ at height $z^{\prime}$ carrying current $i\left(z^{\prime}, t\right)$ are calculated at a general point $P(r, \phi, z)$ by the following equations $[1,5,6]$. These equations representing 


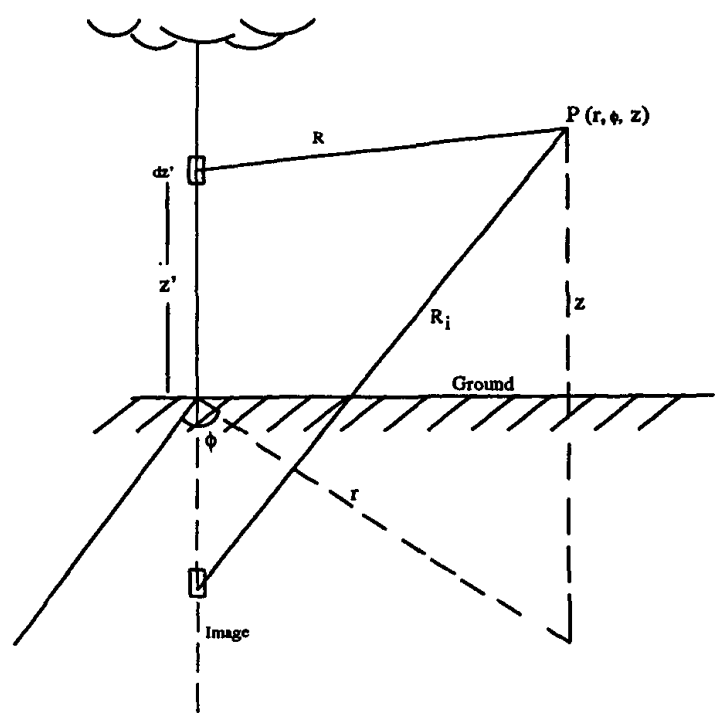

Fig. 1. Sketch showing the lightning channel and its image used in calculating the return stroke fields.

the MTL model for lightning return stroke in time domain are

$$
\begin{gathered}
d E_{z}(r, z, t)=\frac{d z^{\prime}}{4 \pi \epsilon_{0}} \\
{\left[\frac{2\left(z-z^{\prime}\right)^{2}-r^{2}}{R^{5}} e^{\left(-z^{\prime} / \lambda\right)} \int_{0}^{t} i\left(0, \tau-z^{\prime} / v-R / c\right) d \tau\right.} \\
+\frac{2\left(z-z^{\prime}\right)^{2}-r^{2}}{c R^{4}} e^{\left(-z^{\prime} / \lambda\right)} i\left(0, t-z^{\prime} / v-R / c\right) \\
\left.-\frac{r^{2}}{c^{2} R^{3}} e^{\left(-z^{\prime} / \lambda\right)} \frac{\partial}{\partial t} i\left(0, t-z^{\prime} / v-R / c\right)\right] \\
{\left[\frac{3 r\left(z-z^{\prime}\right)}{R^{5}} e^{\left(-z^{\prime} / \lambda\right)} \int_{0}^{t} i\left(0, \tau-z^{\prime} / v-R / c\right) d \tau\right.} \\
\left.+\frac{r\left(z-z^{\prime}\right)}{c^{2} R^{3}} e^{\left(-z^{\prime} / \lambda\right)} \frac{\partial}{\partial t} i\left(0, t-z^{\prime} / v-R / c\right)\right] \\
+\frac{3 r\left(z-z^{\prime}\right)}{c R^{4}} e^{\left(-z^{\prime} / \lambda\right)} i\left(0, t-z^{\prime} / v-R / c\right) \\
4 \pi \epsilon_{0}
\end{gathered}
$$

where $\epsilon_{0}$ is the permittivity of free space and $c$ is the velocity of light. $R=\sqrt{r^{2}+\left(z-z^{\prime}\right)^{2}}$ is the distance from the current element to the observation point. The effects of the perfectly conducting ground plane on the electromagnetic fields generated by the current element are included by replacing the ground by an image current at a distance $z^{\prime}$ below the ground. To find the total field the equations (2) and (3) are integrated along the channel and its image. A sketch showing the lightning channel and its image used in calculating the return stroke fields is shown in figure 1.

\section{Effect of Ground on Horizontal Electric Field}

The horizontal component of electric field produced by the lightning is significantly influenced by the finite conductivity of the ground. The horizontal electric field including the ground conductivity can be computed using Cooray-Rubinstein formula [7]. This formula requires the azimuthal component of the magnetic field produced by the lightning which is given as $[1,6]$.

$d H_{\phi}(r, z, t)=\frac{d z^{\prime}}{4 \pi}\left[\frac{r}{c R^{2}} e^{\left(-z^{\prime} / \lambda\right)} \frac{\partial}{\partial t} i\left(0, t-z^{\prime} / v-R / c\right)\right.$

$$
\left.+\frac{r}{R^{3}} e^{\left(-z^{\prime} / \lambda\right)} i\left(0, t-z^{\prime} / v-R / c\right)\right]
$$

where $\mu_{0}$ and $\sigma_{g}$ are the permeability of free space and the conductivity of the ground respectively.

The horizontal electric field including the ground conductivity $E_{r g}(r, z, t)$ is given as[7]

$E_{r g}(z=h, r)=E_{r}(z=h, r)-H_{\phi}(z=0, r) \frac{\sqrt{\mu_{0}}}{\sqrt{\epsilon+\sigma_{g} / j \omega}}$

where $E_{r}(z=h, r)$ is the Fourier-transform of the horizontal electric field at height $h, H_{\phi}(z=0, r)$ is the Fourier-transform of the azimuthal component of the magnetic field at ground level. $\epsilon$ is the permittivity of the ground. Both $E_{r}(z=h, r)$ and $H_{\phi}(z=0, r)$ are calculated assuming a perfect conducting ground using equations (3) and (4).

\section{Field-to-Transmission Line Coupling Model}

For the field-to-transmission line coupling, a set of time domain differential equations describing the voltages induced as proposed by Agrawal et.al.[8] have been used. The surge propagation is affected by the ground impedance. This is represented in the overhead line parameters as i) per-unit series ground impedance and ii) shunt ground admittance. Both the above quantities are frequency dependent and generally the latter term is neglected. The frequency dependent series ground impedance is represented by a convolution integral in the time-domain coupling equations which are given as 


$$
\begin{gathered}
\frac{\partial}{\partial x}\left[v_{i}^{s}(x, t)\right]+\left[L_{i j}\right] \frac{\partial}{\partial t}\left[i_{i}(x, t)\right] \\
+\int_{0}^{t}\left[\xi_{i j}(t-\tau)\right] \frac{\partial}{\partial \tau}[i(x, \tau)] d \tau=\left[E_{x}^{i}\left(x, h_{i}, t\right)\right] \\
\frac{\partial}{\partial x}\left[i_{i}(x, t)\right]+\left[G_{i j}\right]\left[v_{i}^{s}(x, t)\right]+\left[C_{i j}\right] \frac{\partial}{\partial t}\left[v_{i}^{s}(x, t)\right]=0
\end{gathered}
$$

where $\left[E_{x}^{i}\left(x, h_{i}, t\right)\right]$ is the horizontal component of the incident electric field along the conductor at conductor height $h_{i}$. The sub index $i$ denotes the particular wire of the multiconductor line. $\left[L_{i j}\right],\left[G_{i j}\right]$ and $\left[C_{i j}\right]$ are the inductance, conductance and capacitance matrices per unit length of the line respectively. $\left[i_{i}\right]$ is the line current vector. $\left[\xi_{i j}\right]$ is the transient ground resistance matrix and is equal to the inverse Fourier-transform of $\left[Z_{g_{i j}} / j \omega\right]$, ie., $\xi_{i j}(t)=F^{-1}\left[\frac{Z_{g_{i j}}}{j \omega}\right]$

and $\left[Z_{g_{i j}}\right]$ is the ground impedance matrix. The internal impedance of the line is neglected. $\left[v_{i}^{g}\right]$ is the scattered voltage vector on the $i^{\text {th }}$ conductor. This is the voltage due to the field produced by the induced currents on the conductors. The scattered voltage is related to the total line voltage $v_{i}(x, t)$ by the following relation.

$$
\left[v_{i}(x, t)\right]=\left[v_{i}^{s}(x, t)\right]+\left[v_{i}^{i}(x, t)\right]
$$

where $\left[v_{i}^{i}(x, t)\right]$ is the incident voltage.

$$
\left[v_{i}^{i}(x, t)\right]=-\int_{0}^{h_{i}} E_{z}^{i}(x, z, t) d z
$$

$E_{z}^{i}(x, z, t)$ is the incident(or inducing) vertical electric field at $x$ at a height of $z$.

The voltage at the end of the line is determined by the boundary conditions and the current at the two ends of the line, viz., $i\left(x_{0}, t\right)$ and $i\left(x_{0}+l, t\right)$, where $l$ is the length of the overbead line. The boundary conditions for the scattered voltage are

$$
\begin{gathered}
v_{i}^{s}\left(x_{0}, t\right)=-\left[Z_{1}\right]\left[i_{i}\left(x_{0}, t\right)\right]+\int_{0}^{h_{i}} E_{z}^{i}\left(x_{0}, z, t\right) d z \\
v_{i}^{s}\left(x_{0}+l, t\right)=\left[Z_{2}\right]\left[i_{i}\left(x_{0}+l, t\right)\right]+\int_{0}^{h_{i}} E_{z}^{i}\left(x_{0}+l, z, t\right) d z
\end{gathered}
$$

where $\left[Z_{1}\right]$ and $\left[Z_{2}\right]$ are the terminating impedence matrices. Figure 2 shows the equivalent circuit of this model for a single conductor.

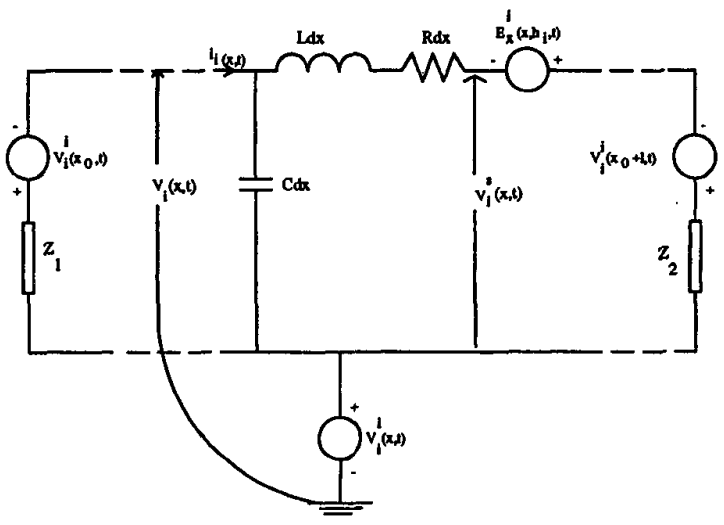

Fig. 2. Equivalent circuit of a single-wire overhead line excited by lightning return-stroke electromagnetic field. Conductance is neglected.

\section{E. Transient Ground Resistance}

In case of multiconductor transmission lines the ground impedance is a full matrix. The low frequency approximation (ie., assuming $\sigma_{g} \gg \omega \epsilon_{0} \epsilon_{r}$ ) for the ground impedance between two conductors $i$ and $j$ is given as [9].

$$
Z_{g_{i j}}=\frac{j \omega \mu_{0}}{\pi} \int_{0}^{\infty} \frac{e^{-\left(h_{i}+h_{j}\right) x} \cos \left(r_{i j} x\right)}{\sqrt{x^{2}+j \omega \mu_{0} \sigma_{g}}+x} d x
$$

where $r_{i j}$ is the distance between the two conductors in the horizontal plane. The low frequency approximation of the inverse Fourier transform of the ground impedance is given by [9].

$$
\begin{gathered}
\xi_{i j}(t)=\frac{\mu_{0}}{\pi T_{i j}}\left[\frac{1}{2 \sqrt{\pi}} \sqrt{\frac{T_{i j}}{t}} \cos \left(\theta_{i j} / 2\right)\right. \\
+\frac{1}{4} e^{T_{i j} \cos \left(\theta_{i j}\right) / t} \cos \left(\frac{T_{i j}}{t} \sin \left(\theta_{i j}\right)-\theta_{i j}\right) \\
\left.-\frac{1}{2 \sqrt{\pi}} \sum_{n=0}^{\infty} a_{n}\left(\frac{T_{i j}}{t}\right)^{\frac{2 n+2}{2}} \cos \left(\frac{2 n-1}{2} \theta_{i j}\right)-\frac{\cos \left(\theta_{i j}\right)}{4}\right]
\end{gathered}
$$

where $a_{n}=\frac{2^{n}}{1.3 \ldots .(2 n+1)}$

with $\left[\frac{h_{i}+h_{i}}{2}+j \frac{r_{i j}}{2}\right]^{2} \mu_{0} \sigma_{g}=T_{i j} e^{j \theta_{i j}}$

In (13), if $h_{i}=h_{j}$ then $r_{i j}=0$ and $\theta_{i j}=0$ we get the expression for diagonal terms of $\left[\xi_{i j}(t)\right]$.

In the above expression, $\xi_{i j}(t)$ tends to infinity as $t \rightarrow 0$. Recently Loyka[10] has shown that $\xi_{i j}(t)$ will tend to be a constant if it is calculated without the low frequency approximation. In the present work, the incremental time $\Delta t$ is selected such that $\xi_{i j}(t)$ is calculated using equation (13). In solving the convolution integral 
the ground transient resistance is taken as constant in the range $t-\Delta t<\tau<t$. Hence for the calculation of line current at $n^{t h}$ incremental time, $t=n \Delta t$, the numerical expression for the convolution integral [11] at any node is obtained as follows,

for $n=2$

$$
\int_{0}^{\Delta t} \xi_{i j}(t-\tau) \frac{\partial}{\partial t} i(x, \tau) d \tau=\xi_{1}(t)\left(i_{1}-i_{0}\right)
$$

for $n>2$

$$
\begin{gathered}
\int_{0}^{(n-1) \Delta t} \xi_{i j}(t-\tau) \frac{\partial}{\partial t} i(x, \tau) d \tau= \\
\sum_{k=1}^{k=(n-2)} \xi_{k}(t)\left(i_{n-k}-i_{n-k-1}\right) \\
+\xi_{1}(t) \frac{\left(i_{n-1}-i_{n-2}\right)}{2}+\xi_{n-1}(t) \frac{\left(i_{1}-i_{0}\right)}{2}
\end{gathered}
$$

with $\xi_{k}(t)$ and $i_{k}$ representing the value of the transient ground resistance for the elements in $\left[\xi_{i j}(t)\right]$ and the line current respectively at time $t=k \Delta t$.

\section{Results and Discussions}

The lightning induced voltages are calculated for $33 \mathrm{kV}, 3 \phi$, single circuit and double circuit overhead power distribution lines. The configuration of the lines are shown in figure 3 . The length of the transmission line is taken as $1 \mathrm{~km}$. The line conductors are terminated at both ends by their characteristic impedance and the earth wire is terminated by the surge impedance of the tower[12]. The induced voltages are calculated at the line ends as well as at the mid point of the line for a lightning stroke located at a distance of $50 \mathrm{~m}$ from the line center and perpendicular to the line for (1) a perfectly conducting ground and (2) with ground conductivity $\sigma_{g}$ of 0.001 $\mathrm{s} / \mathrm{m}$ and relative permittivity $\epsilon_{r}$ of 10 . Figures 4-9 show the induced voltages on conductor 1 at line termination for the various configurations for the following 3 cases.

1. Assuming ground as a perfect conductor - Curve 1.

2. Considering the conductivity of the ground only for the calculation of the horizontal electric field ie., using equation 5 - Curve 2.

3. Considering the conductivity in the calculation of both the horizontal electric field as well as the coupling equations - Curve 3 .

For a lightning return stroke current of $12 \mathrm{kA}$ at ground level, the plots(figures 4, 5 and 6) show that the positive peak of the induced voltage at line terminations for conductor 1 is about 1) $43 \mathrm{kV}$ for single circuit vertical configuration 2) $46 \mathrm{kV}$ for single circuit horizontal configuration and 3) $34 \mathrm{kV}$ for double circuit configuration if
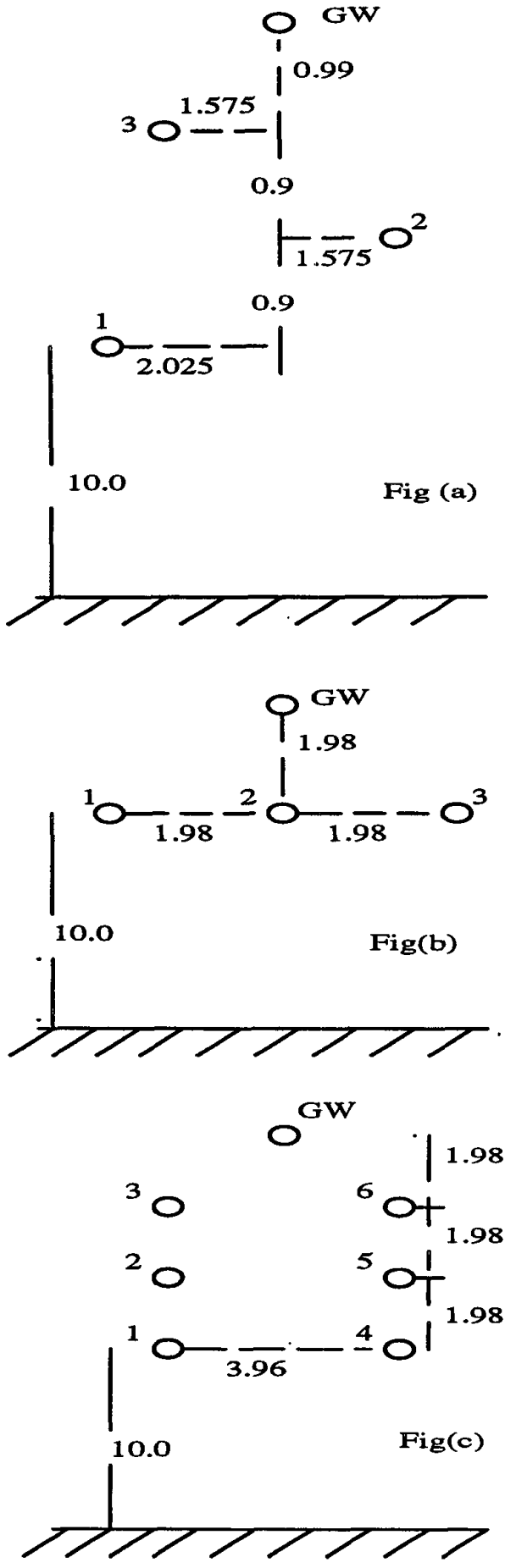

Fig. 3. Conductor configurations used.(a) Single circuit vertical,(b) Single circuit horizontal and (c) Double circuit. GW-ground wire. All dimensions are in meters. 


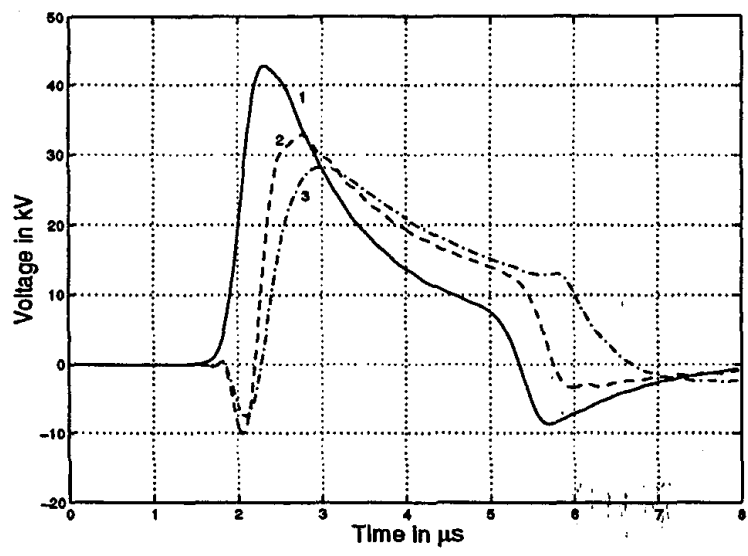

Fig. 4. Induced voltage on conductor 1 of the single circuit vertical configuration at line termination.

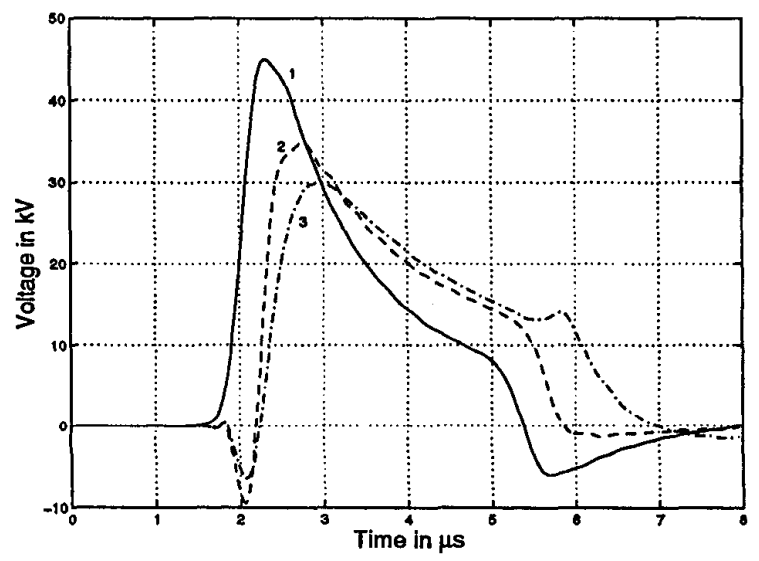

Fig. 5. Induced voltage on conductor 1 of the single circuit horizontal configuration at line termination.

ground is assumed as a perfect conductor. The negative peak of the induced voltages at line terminations are $9 \mathrm{kV}$, $6 \mathrm{kV}$ and $13 \mathrm{kV}$ respectively. Both the positive and negative peaks of the induced voltage get reduced when a finite conductivity is assumed for the ground. The reduction in peaks is much more when ground conductivity is included in the computation of both the horizontal electric field as well as in the coupling to overhead line. There is also an initial negative prepulse in the induced voltage when a finite ground conductivity is included in the computation of horizontal electric field.

Figures 7, 8 and 9 show the induced voltage on conductor 1 at midpoint of the line for the three configurations. At mid point of the line the positive peak magnitude of voltage remains the same at about $80 \mathrm{kV}$, if ground is assumed as a perfect conductor whereas it reaches about $145 \mathrm{kV}$ for a ground conductivity of $0.001 \mathrm{~s} / \mathrm{m}$ and

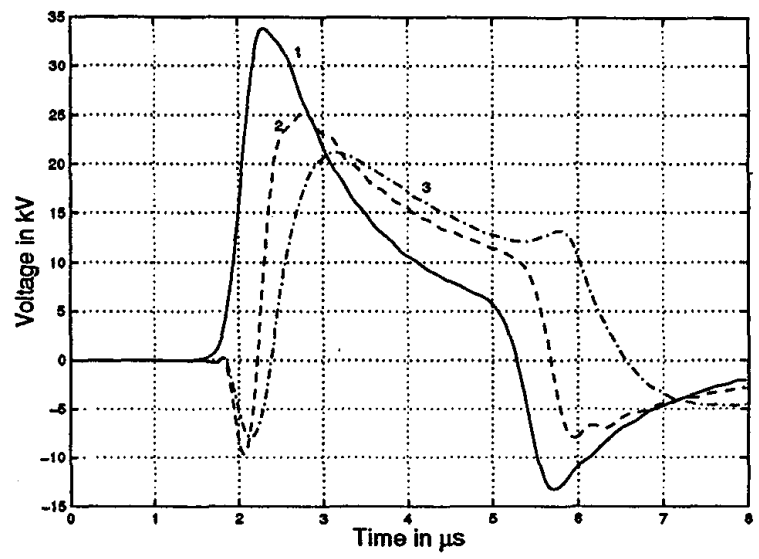

Fig. 6. Induced voltage on conductor 1 of the double circuit configuration at line termination.

relative permittivity of 10 , for all the three configurations studied.

It has been observed from the computation that the magnitude of negative peak voltage is more for double circuit configuration as compared to the other two configurations.The ground conductivity decreases the magnitude of the induced voltage at the line termination where as it increases the voltage at the mid point.

The magnitude and waveshape of the induced voltage mainly depends on the horizontal electric field along the conductor. Since the horizontal electric field has a negative peak initialy due to the finite conductivity of the ground (see figure 10), the voltage induced also posseses initial negative polarity at the line termination where as at the mid point of the line, the horizontal electric field along the direction of the line is zero for the above mentioned stroke location and hence there is no initial negative peak in the induced voltage at the line mid point.

The plots show that on conductor 1, which is at the same height and same distance from strike point in all the configurations, the induced voltage at line termination is less for the double circuit configuration case. This is because when the number of conductors are more, there is a mutual shielding effect coming in to effect there by reducing the induced voltage. Hence the waveshape of the induced voltage on a conductor in multiconductor system is affected by the presence of the other conductors also.

\section{Conclusions}

Induced voltage due to a nearby lightning on $33 \mathrm{kV}, 3 \phi$ single circuit and double circuit power distribution lines have been calculated. The effect of ground conductivity is included in the computation of field as well as in the coupling of field to the overhead conductors. 


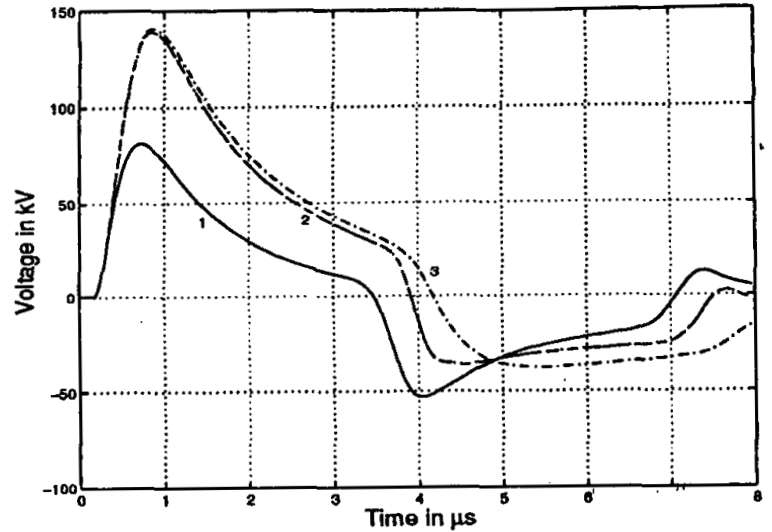

Fig. 7. Induced voltage on conductor 1 of the vertical configuration at mid point of the overhead line.

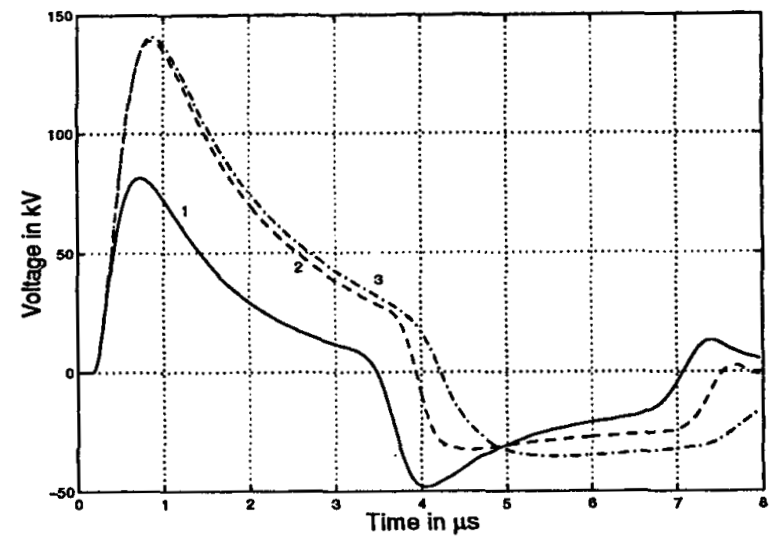

Fig. 8. Induced voltage on conductor 1 of the horizontal configuration at mid point of the overhead line.

When finite ground conductivity is taken in to account, the first peak of induced voltage is reduced at the line termination where as it increases at conductor mid point. The presence of more number of conductors reduces the first peak at line termination.

\section{References}

[1] M.J.Master and M.A.Uman, "Lightning induced voltages on power lines: Theory", IEEE Trans. on Power App.Syst, Vol.PAS-103, No.9 pp 2502-2518, Sept 1984.

[2] S.Guerrieri, C.A.Nucci and F.Rachidi, "Influence of the Ground Resistivity on the Polarity and Intensity of Lightning Induced Voltages", 10th International Symposium on High Voltage Engineering, Canada, pp 199-122, August 1997.

[3] F.Rachidi, C.A.Nucci, M.Ianoz and C.Mazzetti, "Influence of lossy Ground on Lightning Induced Voltages on Overhead Lines", IEEE Trans. Electromag.Compat. Vol 38. No.3, pp 250-264, August 1996.

[4] F.Rachidi, C.A.Nucci and M.Ianoz, "Transient Analysis of Multiconductor Lines Above a Lossy ground", IEEE Trans. Power delivery, vol.14, No.1, pp 294-302, Jan 1999.

[5] C.A.Nucci, F.Rachidi, M.Ianoz and C.Mazzetti, "LightningInduced voltages on overhead Lines", IEEE Trans. Electromag.Compat, Vol.35, No.1, pp 75-85, Feb 1993.

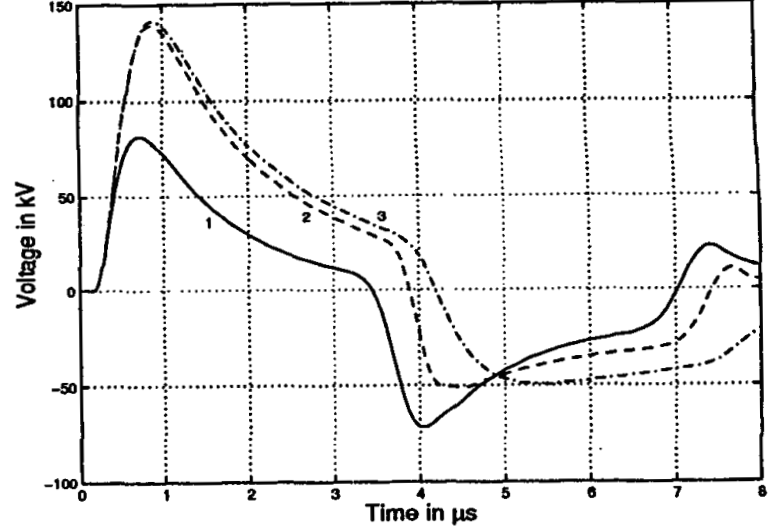

Fig. 9. Induced voltage on conductor 1 of the double circuit configuration at mid point of the overhead line.

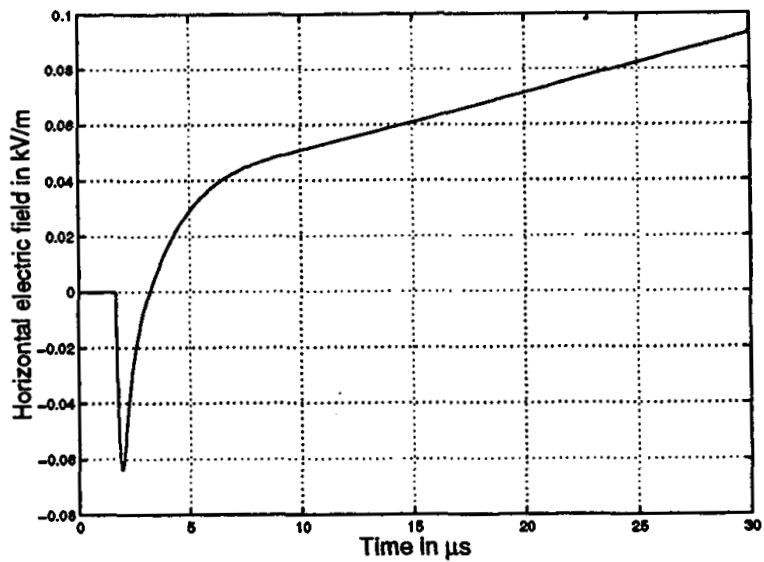

Fig. 10. Horizontal electric field at height $10 \mathrm{~m}$ and distance $500 \mathrm{~m}$ from the lightning stroke for the ground parameters $\sigma_{g}=0.001$ $\mathrm{S} / \mathrm{m}$ and $\epsilon_{\tau}=10$.

[6] M.A.Uman, "Lightning Return Stroke Electric and Magnetic Fields", J1. Geophy.Res, Vol.90, No D4, pp 6121-6130, June 1985.

[7] M.Rubinstein, "An approximate formula for the calculation of the horizontal electric field from lightning at close, intermediate and long ranges", IEEE Trans on EMC, Vol.38, No.3, pp 531-535, August 1996.

[8] A.K.Agrawal, H.J.Price and S.J.Gurbaxani, "Transient Responce of Multiconductor Transmission Lines Excited by a Nonuniform Electromagnetic Field", IEEE Trans on EMC, Vol.22, No.2, pp 119-129, May 1980.

[9] D.Orzan, "Time-domain low frequency approximation for offdiagonal terms of the ground impedance matrix", IEEE Trans. on Electromag.Compat. Vol.39, No.1, p 64, Feb 1997.

[10] S.L.Loyka, "On Calculation of the Ground Transient Re sistance of Overhead Lines", IEEE Trans. on Electromag.Compat. Vol 41. No.3, pp 193-195, August 1999.

[11] E.O.Brigham, "The Fast Fourier Transform", Prentice-Hall, New Jersey Inc. 1974.

[12] Costa, F.S and Miranda, G.C, "Comparison of Lightning Tower Surge Models Using the EMTP", 10th International Symposium on High Voltage Engineering, Canada, pp 327-330, August 1997. 